\title{
What is loop multiplication anyhow?
}

\author{
Joseph A. Neisendorfer ${ }^{1}$
}

Received: 31 January 2016 / Accepted: 17 July 2016 / Published online: 31 August 2016

(C) Tbilisi Centre for Mathematical Sciences 2016

\begin{abstract}
In the beginning we are taught that multiplication of pointed loops is just the first followed by the second. Later we learn that the cobar construction on the chains of the base is a model for the chains on the loop space, that is, this cobar construction is chain equivalent to the chains on the loop space. The cobar construction is a certain tensor algebra and has a natural multiplication of tensors. This multiplication of tensors models the multiplication induced by the multiplication of loops. But, because of the simplicity of the definition of loop multiplication, this modeling is not obvious. We interpret loop multiplication so that this modeling becomes a clear consequence of the naturality of Eilenberg-Moore methods applied to multiple or iterated pullbacks. In contrast, we observe that, if we require that it be invariant under homological equivalence of differential coalgebras, there is no natural modeling of the comultiplication in the loop space. But, in rational homotopy theory, results of Milnor-Moore and Quillen show that there is a natural modeling of the Hopf algebra structure of the loop space.
\end{abstract}

Keywords Loops · Cobar construction

Mathematics Subject Classification Primary 55-02 - 55Q40 · 55Q15; Secondary 55R05

Dedicated to the memories of Sam Gitler and John Moore.

Communicated by Jim Stasheff.

$凶$ Joseph A. Neisendorfer

jnei@math.rochester.edu

1 Department of Mathematics, University of Rochester, Rochester, NY 14625, USA 


\section{Contents}

1 Introduction . . . . . . . . . . . . . . . . . . . . . . . . . 660

2 The cobar construction on a differential graded coalgebra . . . . . . . . . . . . . . 662

3 Cotensor products, pullbacks, and the Eilenberg-Moore theorem . . . . . . . . . . . 663

4 Multiple pullbacks and the extended Eilenberg-Moore theorem . . . . . . . . . . . . 665

5 Loop spaces as pullbacks . . . . . . . . . . . . . . . . . . . . . 666

6 A crucial chain equivalence . . . . . . . . . . . . . . . . . . . . . . . 667

7 Loop multiplication via multiple pullbacks . . . . . . . . . . . . . . . . . . . . . . . . 669

8 Model category structures for differential coalgebras and fibrant objects . . . . . . . . . 671

9 Coalgebra structures in the homology of loop spaces . . . . . . . . . . . . . . . . . . . 674

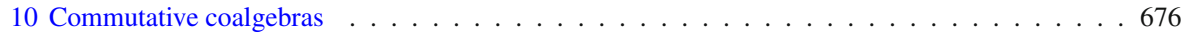

11 Rational loop spaces . . . . . . . . . . . . . . . . . . . . . . . . . . . . . . . 677

12 Quillen's model category of commutative simply connected rational differential graded

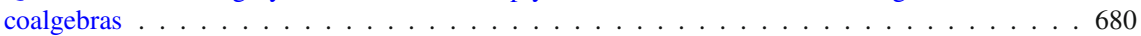

13 Homology of the double loops on spheres . . . . . . . . . . . . . . . . . 682

14 Closing remarks on the Hopf algebra structure in the cobar construction . . . . . . . . . 689

References . . . . . . . . . . . . . . . . . . . . . . . . . . . 689

\section{Introduction}

The multiplication of pointed loops is perhaps the first definition in algebraic topology. Simply run the loops in succession to get a group structure on the fundamental group, that is,

$$
\pi(X) \times \pi(X) \rightarrow \pi(X), \quad(\alpha, \beta) \mapsto \alpha * \beta
$$

where

$$
\begin{gathered}
(\alpha * \beta)(t)=\alpha(2 t), \quad 0 \leq t \leq \frac{1}{2} \\
\beta(2 t-1), \quad \frac{1}{2} \leq t \leq 1
\end{gathered}
$$

is the multiplication of loops.

Let $X$ be a simply connected space and let $C(X)$ be the 1-reduced chains on $X$, that is, there are no non-zero 1-chains. We remark that we always use the normalized chains, that is, the degenerate chains are set equal to 0. We learn from Adams and Hilton $[1,2,14]$ that the cobar construction $\Omega C(X)$ on the chains $C(X)$ is a model for the chains $C(\Omega X)$ on the loop space $\Omega X$. By this, we simply mean that there is a chain equivalence $C(\Omega X) \rightarrow \Omega C(X)$.

There is some debate on exactly what an Adams-Hilton model is for a loop space $\Omega X$ where $X$ is a simply connected space. All authors agree that it is a chain complex constructed as a tensor algebra on some kind of chains on $X$ and that its homology must be that of $\Omega X$.

In the beginning, Adams and Hilton [2] started with a CW complex $X$ and constructed the Adams-Hilton model as the tensor algebra on the CW chains. Adams [1] followed with his explicit cobar construction $\Omega C(X)$ where $C(X)$ are the 1-reduced cubical chains on $X$. Nowadays, the use of cubical chains is rare and the input into the cobar construction is usually the chains on a simplicial set. For example, Hess et al. [14] refer to the cobar construction $\Omega C(X)$ as the canonical Adams-Hilton model where $C(X)$ are the normalized 1-reduced chains on the singular complex of $X$. 
In this paper, we shall refer to this canonical Adams-Hilton model, that is, the cobar construction $\Omega C(X)$, as simply the Adams-Hilton model. One exception to this is in the rational case where the Adams-Hilton model may be taken to be the cobar construction on Quillen's commutative chain complex [25].

The loop multiplication makes the loop space $\Omega X$ into an $\mathrm{H}$-space and, if we use the associative Moore loops, the chains $C(\Omega X)$ become a differential algebra. In the next section we shall see that the cobar construction $\Omega C(X)$ is also a differential algebra. In this note, we shall demonstrate the Adams-Hilton result that the resulting map

$$
\left.H_{*} C(\Omega X)\right) \rightarrow H_{*} \Omega C(X)
$$

is an isomorphism of algebras. We say that the muliplication in the cobar construction or Adams-Hilton model models the multiplication in the chains on the loop space. We shall see that this multiplicative result is a consequence of a definition of loop multiplication via multiple pullback diagrams and of the naturality of an extension of the homological point of view and classical techniques of Eilenberg and Moore [9,23]. We differ from Adams and Hilton in two ways. From the start, we use singular chains instead of $\mathrm{CW}$ chains whereas it is only in the solo sequel that Adams used singular chains. His main motivation was to be able to iterate the construction.

But our most significant departure is that Adams and Hilton need to construct specific maps and then to use the Moore comparison theorem (expose 3 in [6]) to show that it is an equivalence. We require no specific maps here since for us it is a consequence of a generalization of the Eilenberg-Moore methods to multiple or iterated pullbacks. Since we are using Eilenberg-Moore methods, our maps go in the opposite direction than the Adams-Hilton maps go. But these are equivalences so it does not matter.

Getzler and Goerss [10] have given a model structure to the category of differential graded coalgebras but we do not consider this here. We need only the work of Eilenberg-Moore which is prior to the invention of model categories. But that does not mean that there is no connection with model category structures.

In the category of finite type differential comodules over a fixed finite type differential coalgebra, our models for path spaces are fibrant objects. And, when we use Quillen's commutative chains to do rational homotopy theory [25], the fact that the cotensor product is the pullback in this category leads to a coalescing of the Quillen and Eilenberg-Moore points of view.

Even though Baues [3] has shown that, for a simplicial set with a trivial 1-skeleton, this cobar construction has a natural structure of a differential Hopf algebra, we do not concern ourselves here with this coalgebra structure. We only mean that there is an equivalence of chain complexes which we shall show induces a multiplicative isomorphism of homologies.

In fact, given the algebra isomorphism and that the homologies are flat modules, the general theory of Eilenberg-Moore shows that the map

$$
\left.H_{*} C(\Omega X)\right) \rightarrow H_{*} \Omega C(X)
$$

is an isomorphism of Hopf algebras. The point is that the Eilenberg-Zilber map and the Künneth theorem together define a coalgebra structure in the Adams-Hilton model 
$\Omega C(X)$ which agrees with the coalgebra structure in the homology of the loop space $H \Omega X$. But this coalgebra structure cannot be defined simply in terms of the coalgebra $C(X)$. It requires the simplicial structure of the Eilenberg Zilber map which does not exist for an arbitrary differential coalgebra. We show this by considering the case of loops on a suspension.

When $C$ is a differential coalgebra with a commutative and associative comultiplication, then the Adams-Hilton model can be given the structure of a primitively generated differential Hopf algebra. A theorem of Browder [5] then shows that the resulting Hopf algebra structure on $H \Omega C$ is primitively generated. But this coalgebra structure may have little to do with the geometric one on the homology of the loop space $H \Omega X=H \Omega C(X)$ even when there is an equivalence of differential coalgebras $C \rightarrow C(X)$. The actual geometric structure may not be primitively generated.

In the case of rational loop spaces, the work of Milnor-Moore [20,21] and Quillen [25] already showed that the Adams-Hilton model can be given the structure of a primitively generated differential Hopf algebra so that its homology agrees with the homology of the loop space as a Hopf algebra.

In our penultimate section we show how to use the Eilenberg-Moore machinery to compute the mod $p$ homology of the double loop space of a sphere, both the multiplication and the comultiplication, in fact, the entire differential Hopf algebra structure with the Bockstein as differential.

Finally we close with a summary of the overall picture of the Hopf algebra structure in cobar constructions. One goal of this paper is to provide in one place a description of the multiplicative and comultiplicative structures in the homology of Adams-Hilton models applied to differential coalgebras. When do they exist, and when do they agree with geometry? This situation used to confuse me and it still confuses many otherwise competent people. It turns out that the multiplicative structure is always there, it always agrees with geometry, and it is unique. But the comultiplication is much more subtle. It is not always there and, if there, it might not agree with geometry. It is always there in the case of the Adams-Hilton model applied to a simplicial coalgebra and this structure always agrees with geometry. For example, it is always there in the homology of the Adams-Hilton model applied to the chains on a (simply connected) topological space. But this structure is hard to get your hands on since simplicial coalgebras are too large and since the comultiplications in the homology of Adams-Hilton models are not invariant under the input into the Adams-Hilton models of homologically equivalent differential coalgebras. The comultiplication in the homology of the Adams-Hilton models is just not "visible" unless you are given knowledge of something extra such as rational coefficients, or the generating complex in the Bott-Samelson theorem, or the action of Steenrod and other operations, or even just a lucky confinement of the generators to degrees which force primitive generation.

I am grateful to Kathryn Hess for listening to a version of this.

\section{The cobar construction on a differential graded coalgebra}

Let $R$ be a commutative ring and, over $R$, let $C$ be a simply connected differential graded coalgebra with an associative diagonal $\Delta: C \rightarrow C \otimes C$. Thus, $C_{0}=R=$ 
the ground ring and $C_{1}=0$. We have a splitting $C=R \oplus \bar{C}$ where $\bar{C}$ is the part concentrated in (strictly) positive degrees. For any positive degree element $c \in \bar{C}$ we write

$$
\Delta(c)=c \otimes 1+1 \otimes c+\sum c^{\prime} \otimes c^{\prime \prime}=c \otimes 1+1 \otimes c+\bar{\Delta}(c)
$$

where $\bar{\Delta}(c) \in \bar{C} \otimes \bar{C}$.

The cobar construction $\Omega C$ is the tensor algebra $T\left(s^{-1} \bar{C}\right)$ with an internal differential $d_{I}$ and an external differential $d_{E}$. They are both derivations and are given on generators by

$$
d_{I}\left(s^{-1} c\right)=-s^{-1} d c
$$

and

$$
d_{E}\left(s^{-1} c\right)=\sum(-1)^{\operatorname{deg} c^{\prime}}\left(s^{-1} c^{\prime}\right)\left(s^{-1} c^{\prime \prime}\right)
$$

Lemma $2.1 d_{I}^{2}=d_{E}^{2}=0, \quad d_{I} d_{E}=-d_{E} d_{I}$

In the lemma, the first formula is trivial, the second follows from coassociativity of the diagonal, and the third follows from the fact that the diagonal is a coderivation. It follows that the total differential $d_{T}=d_{I}+d_{E}$ has square 0 , that is, it is a differential and it is also a derivation. We define the cobar construction to be the differential graded algebra $\Omega C$ with this total differential $[1,16,23]$.

\section{Cotensor products, pullbacks, and the Eilenberg-Moore theorem}

If $M$ is a right comodule over a coalgebra $C$ and $N$ is a left comodule over $C$, then the cotensor product $M \square_{C} N$ is the kernel of the map

$$
\Delta \otimes 1-1 \otimes \Delta: M \otimes N \rightarrow M \otimes C \otimes N
$$

that is, it is the equalizer of the two maps $\Delta \otimes 1$ and $1 \otimes \Delta$. It is the dual of the tensor product and behaves as one might expect.

Lemma 3.1 (a) $\left(M \square_{C} N\right) \square_{D} P \cong M \square_{C}\left(N \square_{D} P\right)$ when this makes sense and $M, N$ and $P$ are flat modules over the ground ring.

(b) $M \stackrel{\Delta}{\rightarrow} M \otimes C$ and $N \stackrel{\Delta}{\rightarrow} C \otimes N$ are isomorphisms onto $M \square_{C} C$ and $C \square_{C} N$, respectively. In other words, $M=M \square_{C} C$ and $N=C \square_{C} N$ are the isomorphic images of the coactions $\Delta$.

If the ground ring is a field, the above lemma is just the dual of the usual facts about tensor products and, since coalgebras and comodules are direct limits of finite type objects, the lemma follows immediately by dualization. But the above lemma is 
best proved directly. It can be noted that part b) follows from the fact that the standard complex or cobar resolution

$$
0 \rightarrow M \stackrel{\Delta}{\rightarrow} M \otimes C \stackrel{\Delta \otimes 1-1 \otimes \Delta}{\longrightarrow} M \otimes C \otimes C \rightarrow \cdots
$$

is exact $[8,11,18]$.

Given a diagram

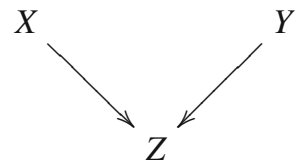

we complete it to a pullback diagram in the form of a diamond

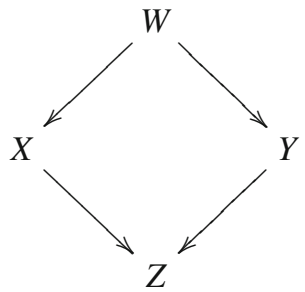

with the pullback denoted by $W=X \times_{Z} Y$, that is, $W$ is the product of $X$ and $Y$ over $Z$.

If $X, Y$, and $Z$ are spaces, the Alexander-Whitney map $\Delta: C W \rightarrow C W \otimes C W$ gives a map $\Delta: C W \rightarrow C X \otimes C Y$. It is clear that this map factors through $\Delta:$ $C W \rightarrow C X \square_{C Z} C Y$.

Recall that a proper resolution is one in which the connectivity of each stage increases by one. This is a strong condition which confines the related assembled total complex to positive degrees.

The main theorem of Eilenberg and Moore [9,23] says

Theorem 3.2 Let $Z$ be simply connected and suppose at least one of $X \rightarrow Z$ or $Y \rightarrow Z$ is a fibration. Let $C X \rightarrow T_{X}$ and $C Y \rightarrow T_{Y}$ be the total complexes of proper injective resolutions of differential comodules over $C Z$, then $\Delta$ composes to give a natural chain equivalence

$$
C W \stackrel{\Delta}{\rightarrow} C X \otimes C Y \rightarrow T_{X} \square_{C Z} T_{Y}
$$

Furthermore, the maps

$$
C X \square_{C Z} T_{Y} \rightarrow T_{X} \square_{C Z} T_{Y} \leftarrow T_{X} \square_{C Z} C Y
$$


are chain equivalences. In fact, any of $C X, C Y$ or $C Z$ can be replaced by a differential objects which are chain equivalent via maps of differential comodules and coalgebras.

The Eilenberg-Moore theorem is often stated in the weaker form that there is an isomorphism of the homology of the pullback with differential Cotor, that is,

$$
H W \stackrel{\cong}{\rightarrow} \operatorname{Cotor}^{C Z}(C X, C Y)
$$

is a natural isomorphism.

The fact that we need only one resolution is referred to by saying that Cotor is a "balanced" functor. The fact that the chain complexes can be replaced by chain equivalent ones is referred to by saying that Cotor is "homologically invariant."

\section{Multiple pullbacks and the extended Eilenberg-Moore theorem}

The Eilenberg-Moore theorem is extended to larger pullback diagrams in the following way. Let $W=X_{1} \times_{A_{1}} X_{2} \times_{A_{2}} X_{3} \times_{A_{3}} \cdots \times_{A_{n-1}} X_{n}$ be the pullback of the diagram

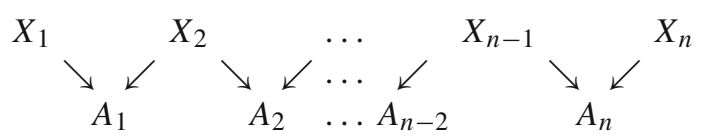

These multiple pullback diagrams are often better understood by expanding them into a collection of iterated "pullback diamonds." We illustrate this in the case $n=3$.

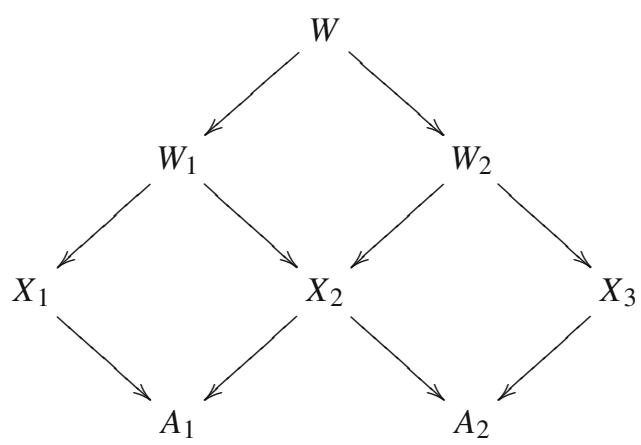

Thus,

$$
W_{1}=X_{1} \times_{A_{1}} X_{2}, \quad W_{2}=X_{2} \times_{A_{2}} X_{3}
$$

and

$$
W=X_{1} \times_{A_{1}} X_{2} \times_{A_{2}} X_{3}=W_{1} \times_{X_{2}} W_{2}
$$


The following extension [23] of the Eilenberg-Moore theorem to multiple pullbacks is easily proved by induction. In fact, in this paper, we use only the cases where $n \leq 3$.

Theorem 4.1 Let $A_{1}, A_{2}, \ldots A_{n-1}$ all be simply connected spaces and suppose that all but possibly one of the maps $X_{i} \rightarrow A_{i}$ and $X_{i} \rightarrow A_{i-1}$ are fibrations. Let $C X_{i} \rightarrow$ $T_{i}$ the total complexes of proper injective resolutions of differential bi-comodules over $C A_{i}$ and $C A_{i-1}$. (At the ends, we do not have bi-comodules but only comodules.) Then $\Delta$ composes to give a natural chain equivalence

$$
C W \rightarrow T_{1} \square_{C\left(A_{1}\right)} T_{2} \square_{C\left(A_{3}\right)} T_{3} \ldots \square_{C\left(A_{n-1}\right)} T_{n}
$$

In particular, the resulting homology isomorphism, denoted

$$
H W \stackrel{\cong}{\rightarrow} \operatorname{Cotor}^{C A_{1}, C A_{2}, \ldots, C A_{n-1}}\left(C X_{1}, C X_{2}, \ldots, C X_{n}\right)
$$

is a natural isomorphism.

And, similar to the case of a simple pullback, we can replace any one $T_{i}$ by $C\left(X_{i}\right)$ or indeed by any comodule which is chain equivalent.

\section{Loop spaces as pullbacks}

If $X$ is a pointed space, let $P_{R} X$ be the space of paths which begin at the basepoint $*$. Then we have a fibration $P_{R} X \rightarrow X$ with fibre the loop space $\Omega X$. Likewise we have a fibration $P_{L} X \rightarrow X$ where $P_{L} X$ is the space of paths which end at the basepoint $*$. Hence we have maps of homotopy equivalent pullback diagrams

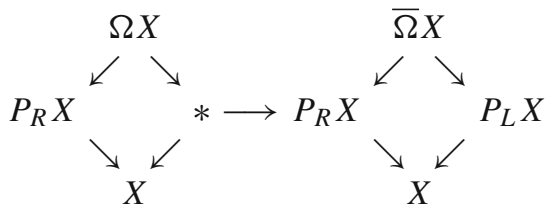

and a similar diagram where the roles of $P_{R} X$ and $P_{L} X$ are interchanged.

Note that the space $\bar{\Omega} X$ is just a pair of paths $(\omega, \sigma)$ such that

$$
\omega(0)=\sigma(1)=*, \quad \omega(1)=\sigma(0) .
$$

This amounts to two paths which connect. Hence, it can be considered to be one path defined on the interval $[0,2]$. The space $\bar{\Omega} X$ is clearly homeomorphic to the usual loop space $\Omega X$.

The map $\tau: C \rightarrow \Omega C$ defined by

$$
\tau c=s^{-1} c, \quad \operatorname{deg} c>0, \quad \tau 1=0
$$


is a "twisting function." That is, if $\Delta$ is the diagonal and $\mu$ is the multiplication, $d \tau+$ $\tau d=\mu(\tau \otimes \tau) \Delta$. Both $\Omega C \otimes C$ and $C \otimes \Omega C$ become acyclic with the new differentials which are the sum of the usual tensor product differential and the respective additions of

$$
D_{\tau}(\alpha \otimes c)=(-1)^{\operatorname{deg} \alpha}\left(\alpha(\tau c) \otimes 1+\alpha\left(\tau c^{\prime}\right) \otimes c^{\prime \prime}\right)
$$

and

$$
D_{\tau}(c \otimes \alpha)=-\left(1 \otimes(\tau c) \alpha+(-1)^{\operatorname{deg} c^{\prime}} c^{\prime} \otimes\left(\tau c^{\prime \prime}\right) \alpha\right)
$$

where $\Delta c=c \otimes 1=1 \otimes c+\sum c^{\prime} \otimes c^{\prime \prime}$.

These acyclic complexes are both the total complexes of resolutions and are denoted respectively by $\Omega C \otimes_{\tau} C$ and $C \otimes_{\tau} \Omega C$.

With $C=C X$, these are both "models" for the chains on the path space, that is, there are chain equivalences

$$
C P_{L} X \simeq C X \otimes_{\tau} \Omega C X, \quad C P_{R} X \simeq \Omega C X \otimes_{\tau} C X
$$

It follows from Eilenberg-Moore that there are natural chain equivalences

$$
C(\Omega X) \rightarrow R \square_{C X} C X \otimes_{\tau} \Omega C X=R \otimes \Omega C X=\Omega C X
$$

and

$$
C(\Omega X) \rightarrow \Omega C X \otimes_{\tau} C X \square_{C X} R=\Omega C X \otimes R=\Omega C X
$$

where $R$ is the ground ring.

The cobar construction $\Omega C X$ is also called the Adams-Hilton model for the loop space.

\section{A crucial chain equivalence}

There is a chain equivalence

$$
C(\bar{\Omega} X) \rightarrow \Omega C X \otimes_{\tau} C X \square_{C X} C(X) \otimes_{\tau} \Omega C X=\Omega C X \otimes_{\tau} C X \otimes_{\tau} \Omega C X .
$$

The differential in the tensor product

$$
\Omega C X \otimes C X \otimes \Omega C X=\Omega C X \otimes_{\tau} C X \otimes_{\tau} \Omega C X
$$

is the unique differential which is compatible with the embedding

$1 \otimes \Delta \otimes 1: \Omega C X \otimes_{\tau} C X \otimes_{\tau} \Omega C X \rightarrow \Omega C X \otimes_{\tau} C X \otimes C X \otimes_{\tau} \Omega C X$. 
Lemma 6.1 The augmentation $\epsilon: C X \rightarrow R$ combined with the multiplication $\mu$ : $\Omega C X \otimes \Omega C X \rightarrow \Omega C X$ gives a chain equivalence

$$
\begin{aligned}
M: \Omega C X \otimes_{\tau} C X \otimes_{\tau} \Omega C X & \stackrel{1 \otimes \epsilon \otimes 1}{\rightarrow} \Omega C X \otimes R \otimes \Omega C X \\
& =\Omega C X \otimes \Omega C X \stackrel{\mu}{\rightarrow} \Omega C X .
\end{aligned}
$$

Proof First we show that $M$ is a chain map. There is a commutative diagram

$$
\begin{array}{ccccc}
\Omega C X \otimes_{\tau} C X \otimes_{\tau} \Omega C X & \stackrel{1 \otimes \epsilon \otimes 1}{\longrightarrow} & \Omega C X \otimes R \otimes \Omega C X & = & \Omega C X \otimes C X \\
\downarrow 1 \otimes \Delta \otimes 1 & & \downarrow 1 \otimes \Delta \otimes 1 & & \downarrow \mu \\
\Omega C X \otimes_{\tau} C X \otimes C X \otimes_{\tau} \Omega C X & 1 \otimes \epsilon \otimes \epsilon \otimes 1 & \Omega C X \otimes R \otimes R \otimes \Omega C X & \stackrel{\text { mult }}{\longrightarrow} & \Omega C X
\end{array}
$$

The map $M$ from the upper left corner down and all the way across is clearly a chain map on the subcomplex $\Omega C X \otimes R \otimes \Omega C X$. We claim it is also a chain map when extended to the whole domain. Let $c \in \bar{C} X$ be a strictly positive degree element. Then $\alpha \otimes c \otimes \beta$ maps to 0 via $M$. To show $M$ is a chain map, it suffices to show that $d(\alpha \otimes c \otimes \beta)$ maps to 0 via $M$.

But $d(\alpha \otimes c \otimes \beta)$ maps down to

$$
d(\alpha \otimes c \otimes 1 \otimes \beta)+d(\alpha \otimes 1 \otimes c \otimes \beta)+d\left(\alpha \otimes c^{\prime} \otimes c^{\prime \prime} \otimes \beta\right) .
$$

The first term is

$d(\alpha) \otimes c \otimes 1 \otimes \beta)+(-1)^{\operatorname{deg} \alpha} \alpha(\tau c) \otimes 1 \otimes 1 \otimes \beta+(-1)^{\operatorname{deg} \alpha} \alpha\left(\tau c^{\prime}\right) \otimes c^{\prime \prime} \otimes 1 \otimes \beta$ $+(-1)^{\operatorname{deg} \alpha+\operatorname{deg} c} \alpha \otimes c \otimes 1 \otimes 0 \beta+(-1)^{\operatorname{deg} \alpha+\operatorname{deg} c} \alpha \otimes c \otimes 1 \otimes d \beta$.

The second term is

$$
\begin{gathered}
d(\alpha) \otimes 1 \otimes c \otimes \beta)+(-1)^{\operatorname{deg} \alpha} \alpha 0 \otimes 1 \otimes c \otimes \beta-(-1)^{\operatorname{deg} \alpha} \alpha \otimes 1 \otimes 1 \otimes(\tau c) \beta \\
-(-1)^{\operatorname{deg} \alpha+\operatorname{deg} c^{\prime}} \alpha \otimes 1 \otimes c^{\prime} \otimes\left(\tau c^{\prime \prime}\right) \beta+(-1)^{\operatorname{deg} \alpha+\operatorname{deg} c} \alpha \otimes 1 \otimes c \otimes d \beta .
\end{gathered}
$$

The third term is a 4 fold tensor with at least one of the middle two tensor of strictly positive degree.

If we now move one step to the right by applying the map $1 \otimes \epsilon \otimes \epsilon \otimes 1$, most terms are annihilated and we get

$$
(-1)^{\operatorname{deg} \alpha} \alpha(\tau c) \otimes 1 \otimes 1 \otimes \beta-(-1)^{\operatorname{deg} \alpha} \alpha \otimes 1 \otimes 1 \otimes(\tau c) \beta .
$$

Finally we apply multiplication which collapses all the tensors, and sends the above to 0 . We get

$$
M d(\alpha \otimes c \otimes \beta)=0
$$

which shows that $M$ is a chain map. 
Second, we claim that $M$ is a chain equivalence.

Since $\Omega C X \otimes_{\tau} C X$ is acyclic, Eilenberg-Moore says that

$$
R \square_{C X} C X \otimes_{\tau} \Omega C X \rightarrow \Omega C X \otimes_{\tau} C X \square_{C X} C X \otimes_{\tau} \Omega C X
$$

is a chain equivalence with $R \rightarrow \Omega C X \otimes_{\tau} C X$ given by $1 \mapsto 1 \otimes 1$. That is,

$$
R \otimes \Omega C X \rightarrow \Omega C X \otimes_{\tau} C X \otimes_{\tau} \Omega C X
$$

is a chain equivalence with $1 \otimes \alpha \mapsto 1 \otimes 1 \otimes \alpha$. Since the composition

$$
\Omega C X=R \otimes \Omega C X \rightarrow \Omega C X \otimes_{\tau} C X \otimes_{\tau} \Omega C X \stackrel{M}{\rightarrow} \Omega C X
$$

is the identity, it follows that $M$ is a chain equivalence.

Alternatively [23], one can check that the differential in $\Omega C X \otimes_{\tau} C X \otimes_{\tau} \Omega C X$ is given by

$$
d=d_{\tau} \otimes 1+1 \otimes d_{\tau}-1 \otimes d_{C X} \otimes 1
$$

where $d_{\tau}$ denotes the differential on the appropriate twisted tensor product. This amounts to showing that the following diagram commutes

$$
\begin{gathered}
\Omega C X \otimes_{\tau} C X \otimes_{\tau} \Omega C X \stackrel{1 \otimes \Delta \otimes 1}{\longrightarrow} \Omega C X \otimes_{\tau} C X \otimes C X \otimes_{\tau} \Omega C X \\
\downarrow d_{\tau} \otimes 1+1 \otimes d_{\tau} \quad \downarrow d_{\tau} \otimes 1 \otimes 1 \\
-1 \otimes d_{C X} \otimes 1 \quad+1 \otimes 1 \otimes d_{\tau} \\
\Omega C X \otimes_{\tau} C X \otimes_{\tau} \Omega C X \stackrel{1 \otimes \Delta \otimes 1}{\longrightarrow} \Omega C X \otimes_{\tau} C X \otimes C X \otimes_{\tau} \Omega C X .
\end{gathered}
$$

Given the explicit formula for the differential, it is easy to see that $M$ is a chain map. Remark In the above lemma, the signs involved in the differential in $\Omega C X \otimes_{\tau} C X \otimes_{\tau}$ $\Omega C X$ are crucial. Nothing would work without them.

\section{Loop multiplication via multiple pullbacks}

In terms of multiple pullbacks, loop multiplication

$$
\Omega X \times \Omega X \rightarrow \bar{\Omega} X, \quad(\omega, \sigma) \mapsto(\omega, \sigma) \equiv \omega * \sigma
$$


is given by the map of the top diagram to the diagram below it
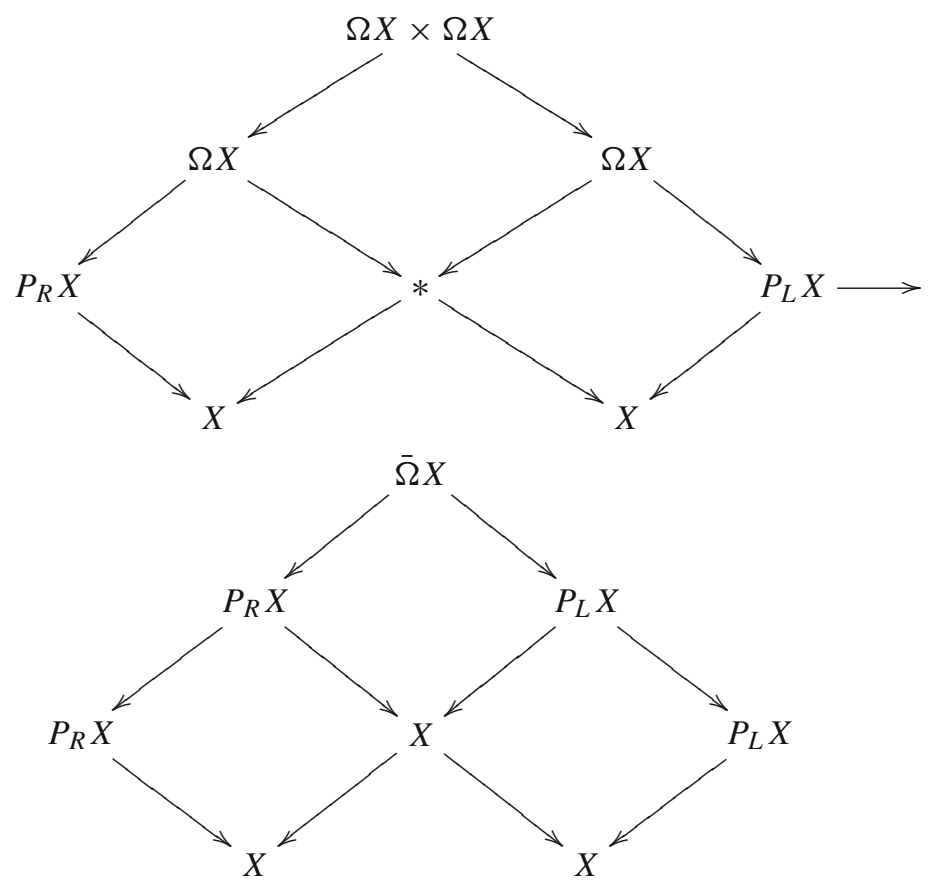

When we apply the chain functor, the diagrams are chain equivalent to
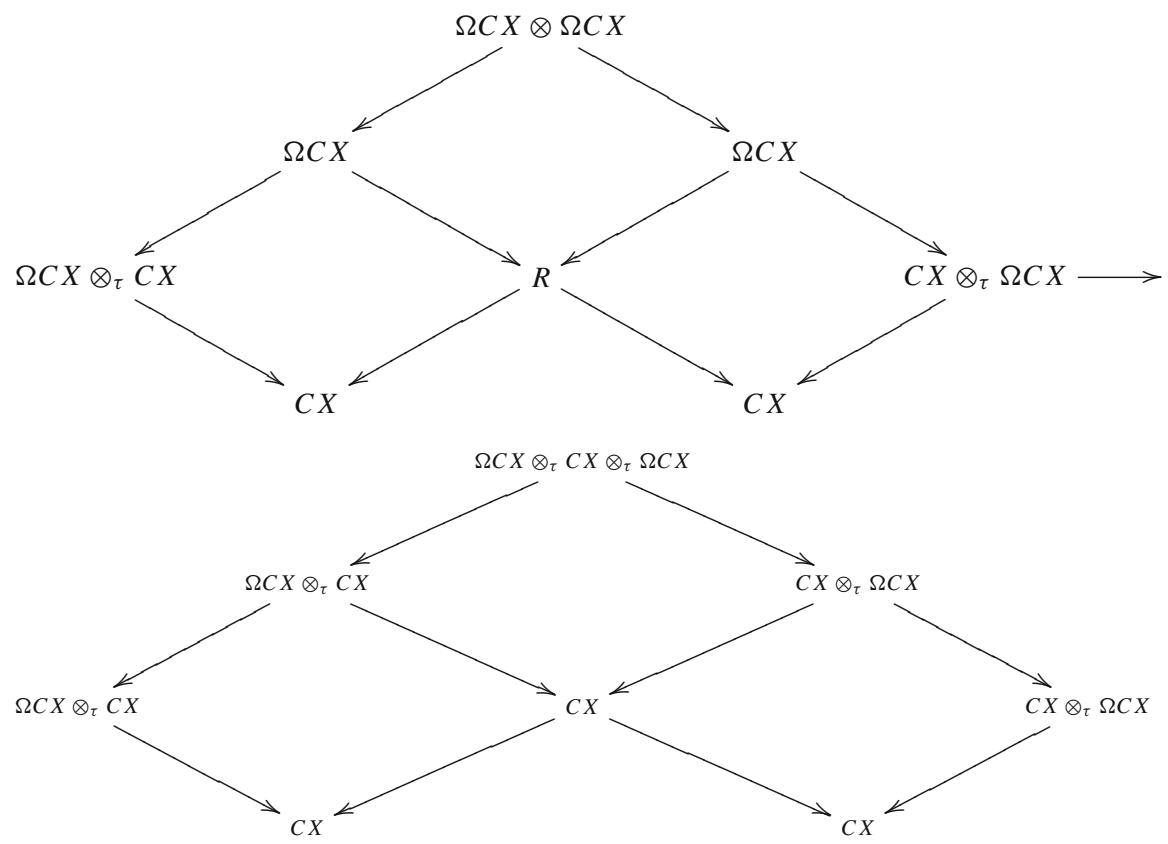
In these terms, the loop multiplication induces the chain map

$$
\begin{aligned}
& \Omega C X \otimes \Omega C X=\Omega C X \otimes R \otimes \Omega C X \rightarrow \Omega C X \otimes_{\tau} C X \otimes_{\tau} \Omega C X, \\
& \alpha \otimes \beta \mapsto \alpha \otimes 1 \otimes \beta .
\end{aligned}
$$

This composes with the chain equivalence $M$ to show that loop multiplication is modeled by the tensor multiplication in the cobar construction

$$
\Omega C X \otimes \Omega C X \rightarrow \Omega C X \otimes_{\tau} C X \otimes_{\tau} \Omega C X \stackrel{M}{\rightarrow} \Omega C X, \quad \alpha \otimes \beta \mapsto \alpha \beta
$$

That is, if $H \Omega C$ is flat over $R$, then loop multiplication induces in homology the map

$$
\begin{aligned}
H(\Omega C X) \otimes H(\Omega C X) & \rightarrow H(\Omega C X \otimes \Omega C X) \rightarrow H\left(\Omega C X \otimes_{\tau} C X \otimes_{\tau} \Omega C X\right) \\
& \stackrel{M}{\rightarrow} H \Omega C X .
\end{aligned}
$$

If $H(\Omega X)$ is flat over $R$, the first map is an isomorphism. The map $M$ is always an isomorphism.

We close with the following remark. Let $C$ be a simply connected differential coalgebra over a commutative ring $R$. There is a homology isomorphism $H \Omega C \cong$ $\operatorname{Cotor}^{C}(R, R)$. In the Eilenberg-Moore language of differential Cotor extended to several variables, the tensor multiplication in the cobar construction $\Omega C \otimes \Omega C \rightarrow \Omega C$ induces in homology the map

$$
\begin{aligned}
\operatorname{Cotor}^{C}(R, R) \otimes \operatorname{Cotor}^{C}(R, R) & \rightarrow \operatorname{Cotor}^{C, C}(R, R, R) \rightarrow \operatorname{Cotor}^{C, C}(R, C, R) \\
& =\operatorname{Cotor}^{C}(R, R) .
\end{aligned}
$$

The first arrow is an isomorphism if $\operatorname{Cotor}^{C}(R, R)$ is flat over $R$. This multiplication is natural with respect to morphisms of the differential coalgebra $C$.

We refer the reader to [23] for further details on differential Cotor of several variables. The main point is that differential Cotor is defined by taking cotensor products of the assembled complexes associated to resolutions of the comodules. And, as in the two variable case, one can omit resolving any one of the comodule variables. In particular, we refer to Section 10.22 of [23] for the proof that, whenever either or both of $H \Omega C$ and $H \Omega D$ are flat over $R$, then the Kunneth map

$$
H \Omega C \otimes H \Omega D \rightarrow H \Omega(C \otimes D)
$$

is an isomorphism of algebras.

\section{Model category structures for differential coalgebras and fibrant objects}

Let $C$ be a differential coalgebra over a commutative ring $R$ and consider the category ${ }_{C} \mathbf{C}$ of (left) differential comodules over $C$. 
Suppose that $C$ is simply connected, that is, $C_{0}=R, C_{1}=0$. If the ground ring $R$ is a field and both the coalgebra and the comodules are assumed to be of finite type then Kathryn Hess [12] has shown that there is a model category structure on ${ }_{C} \mathbf{C}$ in which the weak equivalences are homology isomorphisms, the cofibrations are degreewise injections, and the fibrations are all morphisms which have the right lifting property with respect to all acyclic cofibrations. That is, $p: E \rightarrow B$ is a fibration if and only if the commutative rectangular diagram below with $i: N \rightarrow M$ a cofibration and a weak equivalence, can always be extended as below to a commutative diagram which includes the slanted arrow

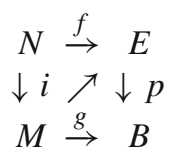

We remark that the original Quillen definition of model category is used. [24] Only finite limits and finite colimits are required.

Later Kathryn Hess and Brooke Shipley showed that this statement is true if $C$ is required to be simply connected, finite type and free in each degree, the ground ring $R$ is required to be semi-hereditary, but now comodules are not restricted to be finite type. [15] Finally, the team of Hess, Kedziorek. Riehl, and Shipley achieved the desired goal of proving it without any assumptions of simple connectivity, finite type, freeness, and over any commutative ring [13].

In the original case considered by Kathryn Hess, we show that Cartan's constructions give examples of fibrant objects.

Recall that a construction $D$ in the sense of Cartan is a differential comodule over $C$ which satisfies: $D=C \otimes X$ as a comodule when we forget the differential, that is, the coaction is

$$
\Delta: D=C \otimes X \stackrel{\Delta \otimes 1}{\longrightarrow} C \otimes C \otimes X=C \otimes D
$$

and the coaction is a chain map.

If $C$ is a differential coalgebra, $A$ is a differential algebra, and $\tau: C \rightarrow A$ is a twisting function, then the twisted tensor product $C \otimes_{\tau} A$ is a an example of a construction.

If the unique map $D \rightarrow 0$ is a fibration, then $D$ is called a fibrant object. Hence, $D$ is fibrant if and only if, whenever $i: N \rightarrow M$ is an acyclic cofibration (= cofibration and weak equvalence), then any map $N \rightarrow D$ extends to a map $M \rightarrow D$.

We conjecture that a Cartan construction $D$ is always a fibrant object.

Unfortunately we can only prove this in the category of differential comodules over a field where the differential coalgebra is finite type and simply connected.

Theorem 8.1 Let $C$ be a simply connected differential coalgebra over a field. Let $D$ be a finite type construction over $C$. Then $D$ is fibrant in the model category of finite type differential comodules. That is, if $i: N \rightarrow M$ is an acyclic cofibration with domain and range both of finite type, then any map $N \rightarrow D$ extends to a map $M \rightarrow D$. 
Proof With the hypotheses we have, it is sufficient to prove the dual statement for differential modules over a simply connected differential algebra $A$ with differentials $\delta$ of degree +1 . The appropriate dual to a Cartan comodule construction is a differential $A$ module $D$ which is isomorphic to $A \otimes K$ when we forget the differential. The dual of our extension problem is the lifting problem

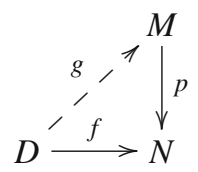

where $g: D \rightarrow M$ is to be constructed as a lift of $f: D \rightarrow N$ given that $p: M \rightarrow N$ is a fibration (= epimorphism) and a weak equivalence (= homology isomorphism).

We begin by decomposing the differential module $D / \bar{A} D \cong K$ into a direct sum of its homology and an acyclic summand. This shows that each $K^{n}$ can be chosen to have a basis $\left\{x_{\alpha}, x_{\beta}, x_{\gamma}\right\}$ where

$$
\delta x_{\alpha}=\sum a_{i} \otimes k_{i}, \begin{array}{lc}
\quad \operatorname{deg} a_{i} \geq 2, \operatorname{deg} k_{i} \leq n-1 & \text { is decomposable } \\
\delta x_{\beta}=y_{\beta} & \text { is a basis element } \in K^{n+1} \\
x_{\gamma}=\delta z_{\gamma} & \text { where z is a basis element } \in K^{n-1}
\end{array}
$$

Then

$$
K[n]=K^{\leq n-1} \oplus\left\langle x_{\alpha}, x_{\beta}, \delta x_{\beta}, x_{\gamma}\right\rangle
$$

Note that $D$ has a complete ascending filtration by subdifferential modules $A \otimes K[n]$. We make the inductive assumption that a module chain lift $g$ of $f$ has been constructed over $A \otimes K[n-1]$ and seek to extend it to $A \otimes K[n]$.

First $g\left(x_{\gamma}\right)$ is defined by $g\left(x_{\gamma}\right)=g\left(\delta z_{\gamma}\right)=\delta g\left(z_{\gamma}\right)$. Since $p g\left(z_{\gamma}\right)=f z_{\gamma}$,

$$
p g\left(x_{\gamma}\right)=p \delta g\left(z_{\gamma}\right)=\delta p g\left(z_{\gamma}\right)=\delta f\left(z_{\gamma}\right)=f\left(\delta z_{\gamma}\right)=f\left(x_{\gamma}\right) .
$$

We note that $p$ is an epimorphism on both cocycles and coboundaries.

Choose a coboundary $w_{\beta} \in M^{n+1}$ so that $p w_{\beta}=f y_{\beta}=f\left(\delta x_{\beta}\right)=\delta f x_{\beta}$. Define $g y_{\beta}=w_{\beta}$.

There is an element $z_{\beta}$ so that $\delta z_{\beta}=w_{\beta}$. Define $\bar{g} x_{\beta}=z_{\beta}$. Then

$$
\delta p \bar{g} x_{\beta}=p \delta \bar{g} x_{\beta}=p \delta z_{\beta}=p w_{\beta}=f y_{\beta}=f \delta x_{\beta}=\delta f x_{\beta} .
$$

Thus $p \bar{g} x_{\beta}-f x_{\beta}$ is a cocycle. Choose a cocycle $u_{\beta}$ so that $p u_{\beta}=p \bar{g} x_{\beta}-f x_{\beta}$.

Set $g x_{\beta}=\bar{g} x_{\beta}-u_{\beta}$. Then $p g x_{\beta}=f x_{\beta}$ and note that

$$
\delta g x_{\beta}=\delta z_{\beta}=w_{\beta}=g y_{\beta}=g\left(\delta x_{\beta}\right)
$$

Of course, $g\left(y_{\beta}\right)=g\left(\delta x_{\beta}\right)=\delta g\left(x_{\beta}\right)$. 
Finally, note that $g\left(\delta x_{\alpha}\right)$ is already defined and it is a cocycle. Furthermore, $p g\left(\delta x_{\alpha}\right)=f\left(\delta x_{\alpha}\right)=\delta f x_{\alpha}$. Since $p g\left(\delta x_{\alpha}\right)$ is a coboundary, there is an element $z_{\alpha}$ so that $\delta z_{\alpha}=g\left(\delta x_{\alpha}\right)$. Define $\bar{g} x_{\alpha}=z_{\alpha}$. Then

$$
\delta p \bar{g} x_{\alpha}=p \delta \bar{g} x_{\alpha}=p \delta z_{\alpha}=p g\left(\delta x_{\alpha}\right)=f \delta x_{\alpha}=\delta f x_{\alpha} .
$$

Thus $p \bar{g} x_{\alpha}-f x_{\alpha}$ is a cocycle. Choose a cocycle $u_{\alpha}$ so that $p u_{\alpha}=p \bar{g} x_{\beta}-f x_{\alpha}$.

Set $g x_{\alpha}=\bar{g} x_{\alpha}-u_{\alpha}$. Then $p g x_{\alpha}=f x_{\alpha}$ and

$$
\delta g x_{\alpha}=\delta \bar{g}_{\alpha}-\delta u_{\alpha}=\delta \bar{g} x_{\alpha}=\delta z_{\alpha}=g\left(\delta x_{\alpha}\right) .
$$

Hence, a lift $g$ of $f$ has been defined on $K[n]$ compatible with the differential and it can be uniquely extended to a lift $g: A \otimes K[n] \rightarrow M$ by making it a module map, that is, $g(a \otimes x)=a(g x)$. It is easy to check that this $g$ is a chain map which lifts $f$.

In a model category a fibrant model for $E$ is a weak equivalence $E \rightarrow D$ with $D$ fibrant. The axioms for a model category give fibrant models since they guarantee the existence of a factorization $E \stackrel{i}{\rightarrow} D \stackrel{p}{\rightarrow} 0$ with $i$ an acyclic cofibration and $p$ a fibration. In particular, when $C$ is a simply connected finite type differential coalgebra over a field, the algebraic model for the path space $R \rightarrow C \otimes_{\tau} \Omega C$ is a fibrant model in the category of finite type differential comodules.

\section{Coalgebra structures in the homology of loop spaces}

In this section, it is essential that we use the normalized chains on a topological space, that is, the chains in which degenerate simplices are set to zero.

The Eilenberg-Zilber map $\nabla: C(X) \otimes C(Y) \rightarrow C(X \times Y)$ is a map of differential coalgebras. The Alexander-Whitney map $\Delta: C(X \times Y) \rightarrow C(X) \otimes C(Y)$ and the Alexander-Whitney diagonal $\Delta: C(X) \rightarrow C(X \times X) \rightarrow C(X) \otimes C(X)$ are not. Hence, in general, the Alexander-Whitney diagonal does not define a map of AdamsHilton models $\Omega C(X) \rightarrow \Omega(C(X) \otimes C(X))$, But recall $[9,23]$

Theorem 9.1 (a) The Eilenberg-Zilber map $\nabla: C(X) \otimes C(Y) \rightarrow C(X \times Y)$ is a chain equivalence.

(b) There is a Künneth map $\Omega C \otimes \Omega D \rightarrow \Omega(C \otimes D)$ which is a chain equivalence.

If the homology $H \Omega X$ is flat over the ground ring, then a diagonal is defined by the composition

$$
\begin{aligned}
\Delta: H \Omega C(X) \stackrel{\Omega \Delta}{\longrightarrow} H(\Omega C(X \times X)) \stackrel{\Omega \nabla}{\longleftarrow} H(\Omega(C(X) \otimes C(X))) \\
\cong H(\Omega C(X)) \otimes H(\Omega C(X)) .
\end{aligned}
$$

This diagonal makes $H \Omega C(X)$ into a Hopf algebra isomorphic to the Hopf algebra $H \Omega X$. 
The definition of this diagonal depends on the fact that $C(X)$ is not just any differential coalgebra but is the chains on a space or simplicial set. For an arbitrary differential coalgebra, it does not exist.

There is no way to define a coalgebra structure in the homology of an Adams Hilton model $\Omega C$ so that two things are satisfied. One, if $C \rightarrow D$ is a morphism of differential coalgebras which is a homology equivalence, then the induced map $\Omega C \rightarrow \Omega D$ should be an isomorphism of coalgebras. We already know that it is an isomorphism of algebras. Two, if $C=C(X)=$ the chains on a space $X$, then there should be isomorphism of coalgebras $H \Omega C \cong H \Omega X$. We already know that it is an isomorphism of algebras.

In short, there is no homologically invariant way to define a coalgebra structure in the cobar construction which will be compatible with geometry.

To see this, start with

Theorem 9.2 If $X$ is a connected space and the homology $H X$ is free over the ground ring, then there is a homology isomorphism of differential coalgebras $H \Sigma X \rightarrow$ $C(\Sigma X)$

Proof Let $Y$ be any connected simplicial set with only one element in degree 0 . Form the simplical cone $y * Y$ and the simplicial suspension $y * Y / Y$. Then it is easy to see that every element of positive degree is primitive under the Alexander-Whitney diagonal $\Delta: C(y * Y / Y) \rightarrow C(y * Y / Y) \otimes C(y * Y / Y)$. In other words, $C(y * Y / Y)$ is a coalgebra with a trivial coproduct. If $H Y$ is free, we can pick a basis of cycles which defines an equivalence of differential coalgebras $H(\Sigma Y)=H(y * Y / Y) \rightarrow C(y * Y / Y)$.

Let $Y=S X=$ the singular chains on a space $X$. We can assume that there is only one zero simplex. Note that there is an embedding $C(y * Y / Y) \subset C \Sigma X$ which is an equivalence. Composition gives an equivalence of differential coalgebras $H \Sigma X \rightarrow C(y * Y / Y) \rightarrow C \Sigma X$.

Corollary 9.3 If $X$ is connected then $H \Omega \Sigma X=\Omega \Sigma H X=T(\bar{H} X)$ as algebras.

Of course, the Bott-Samelson theorem [4] says more. In fact, $H \Omega \Sigma X=\Omega \Sigma H X=$ $T(\bar{H} X)$ as Hopf algebras where the diagonal is determined by the map $X \rightarrow \Omega \Sigma X$.

Let $Z=\Sigma C P^{\infty}$ and let $W=\bigvee_{n=1}^{\infty} S^{2 n+1}$. The coalgebras $H Z$ and $H W$ are isomorphic trivial coalgebras but the homologies of the loop spaces are isomorphic as algebras and not as Hopf algebras.

$$
H \Omega Z=T\left(x_{1}, x_{2}, x_{3}, \ldots\right), \quad H \Omega W=T\left(y_{1}, y_{2}, y_{3}, \ldots\right)
$$

where $\operatorname{deg}\left(x_{n}\right)=\operatorname{deg}\left(y_{n}\right)=2 n$, the $y_{2 n}$ are all primitive, but

$$
\Delta x_{n}=1 \otimes x_{n}+x_{1} \otimes x_{n-1}+\cdots+x_{n-1} \otimes x_{1}+x_{n} \otimes 1
$$

In the first three nontrivial dimensions, a basis for the integral primitives of $H \Omega Z=$ $T\left(x_{1}, x_{2}, x_{3}, \ldots\right)$ is

$$
x_{1}, \quad 2 x_{2}-x_{1}^{2}, \quad x_{2} x_{1}-x_{1} x_{2}, \quad 3 x_{3}-3 x_{2} x_{1}+x_{1}^{3} .
$$


Hence, $H \Omega Z$ is not primitively generated but $H \Omega W$ is.

On the other hand, over the rationals, a theorem of Milnor and Moore shows that all connected Hopf algebras with commutative and associative diagonal are primitively generated [20,21]. Hence, these Hopf algebras are isomorphic over the rationals.

\section{Commutative coalgebras}

Let $\tau: C \rightarrow A$ and $\sigma: D \rightarrow B$ be twisting functions. Define

$$
\tau \boxplus \sigma: C \otimes D \rightarrow A \otimes B
$$

to be $\tau \otimes 1$ on $C \otimes 1,1 \otimes \sigma$ on $1 \otimes D$, and 0 on the standard complement to $C \boxplus D=C \otimes 1+1 \otimes D$ in $C \otimes D$.

Then it is straightforward to show

Lemma 10.1 (a) $\tau \boxplus \sigma: C \otimes D \rightarrow A \otimes B$ is a twisting function.

(b) The tensor product of twisted tensor products is a twisted tensor product, that is,

$$
\left(C \otimes_{\tau} A\right) \otimes\left(D \otimes_{\sigma} B\right) \equiv(C \otimes D) \otimes_{\tau \boxplus \sigma}(A \otimes B) .
$$

It is equally straightforward to show

Lemma 10.2 Let $C$ is a differential coalgebra with a commutative diagonal.

(a) Then the diagonal $\Delta: C \rightarrow C \otimes C$ is a morphism of differential coalgebras.

(b) If we define a coalgebra structure in the cobar construction $\Omega C=T\left(s^{-1} \bar{C}\right)$ by making the generators $s^{-1} \bar{C}$ primitive, that is,

$$
\Delta\left(s^{-1} c\right)=s^{-1} c \otimes 1+1 \otimes s^{-1} c
$$

then the cobar construction becomes a differential Hopf algebra.

Recall a theorem of Browder [5].

Theorem 10.3 If $A$ is a primitively generated Hopf algebra over a field, then the homology Hopf algebra $H A$ is also primitively generated.

Browder actually stated this theorem only for Hopf algebras of finite type but, since every primitively generated Hopf algebra is a direct limit of primitively generated Hopf algebras of finite type, the general case follows.

Hence,

Theorem 10.4 With this Hopf algebra structure over a field, the homology $H \Omega C$ is a primitively generated Hopf algebra whenever $C$ is a commutative differential coalgebra. 
We do not assert that the above is the Hopf algebra structure on the homology $H \Omega X$ of the loop space even when $X$ is a simply connected space, $C=H X$, and there is a homology equivalence of differential coalgebras $H X \rightarrow C X$. Of course the algebra structures agree.

Let $C$ and $D$ be commutative differential coalgebras, let $A$ and $B$ be differential algebras, and let $\tau: C \rightarrow A$ and $\sigma: D \rightarrow B$ be twisting functions.

It is easy to check that:

Lemma 10.5 If $f: C \rightarrow D$ and $g: A \rightarrow B$ are morphisms which commute with the twisting functions, that is, $g \circ \tau=\sigma \circ f$, then $f \otimes g: C \otimes_{\tau} A \rightarrow D \otimes_{\sigma} B$ is a map of differential comodules with respect to the map $f: C \rightarrow D$.

An immediate corollary is

Corollary 10.6 If $C$ is a differential coagebra with a commutative diagonal, then the diagonal maps define a map

$$
\Delta \otimes \Delta: C \otimes_{\tau} \Omega C \rightarrow(C \otimes C) \otimes_{\tau \boxplus \tau}(\Omega C \otimes \Omega C)
$$

of algebraic path spaces which is a map of differential comodules with respect to the map $\Delta: C \rightarrow C \otimes C$.

When $C$ is a commutative differential coalgebra over the ground ring $R$ and $H \Omega C$ is flat over the ground ring, the above has the consequence $[9,16,23]$ that the there is an isomorphism of Hopf algebra structures

$$
H \Omega C \equiv \operatorname{Cotor}^{C}(R, R) .
$$

Now suppose 2 is a unit in the ground ring and that $C$ is a differential coalgebra with a commutative diagonal. Then Quillen observed that the cobar construction $\Omega C=$ $T\left(s^{-1} \bar{C}\right)=U L\left(s^{-1} \bar{C}\right)$ is the universal enveloping algebra of the differential Lie algebra $L=L\left(s^{-1} \bar{C}\right)$ where $L$ is primitive and is the free Lie algebra generated by the desuspension of the reduced coalgebra $\bar{C}$. In terms of $\mathrm{L}$, the differential is given by

$$
d\left(s^{-1} c\right)=-s^{-1} d c+(1 / 2) \sum(-1)^{\operatorname{deg} c^{\prime}}\left[s^{-1} c^{\prime}, s^{-1} c^{\prime \prime}\right]
$$

where

$$
\Delta c=c \otimes 1+1 \otimes c+\sum c^{\prime} \otimes c^{\prime \prime}
$$

\section{Rational loop spaces}

The theorem of Milnor and Moore [19-21] is decisive for the rational homology of loop spaces 
Theorem 11.1 Over a field of characteristic zero, every connected Hopf algebra A with commutative and associative diagonal is primitively generated and is isomorphic to the universal enveloping algebra on its Lie algebra $P(A)$ of primitives, that is,

$$
U P(A) \stackrel{\cong}{\rightrightarrows} A .
$$

Moreover, if $A=H Y$ where $Y$ is a connected group-like space, that is, a connected homotopy associative $H$-space with a homotopy inverse, then the rational Hurewicz map is an isomorphism of the rational homotopy Lie algebra $(\pi Y) \otimes Q$ onto $P H Y$.

The second part of this theorem follows from results of Cartan and Serre on the vanishing of rational $\mathrm{k}$ invariants of $\mathrm{H}$-spaces. Hence, the rational Hurewicz map is an isomorphism $(\pi Y) \otimes Q \rightarrow P H Y$ and Milnor-Moore gives an isomorphism of rational Hopf algebras $U(\pi Y) \otimes Q \rightarrow H Y$.

We conclude by pointing out, as did Quillen [25], that the result of Milnor and Moore fits into and can be derived from his rational homotopy theory.

If $C^{N}=C$ denotes the normalized chains, then the diagonal map on the chains of the loop space

$$
C^{N}(\Delta): C^{N}(\Omega X) \rightarrow C^{N}(\Omega X \times \Omega X)
$$

induces in homology the map of differential Cotors: (See 10,16.2, also 10.21.2, [23].)

$$
\operatorname{Cotor}^{\Delta}(R, R): \operatorname{Cotor}^{C^{N}(X)}(R, R) \rightarrow \operatorname{Cotor}^{C^{N}(X \times X)}(R, R)
$$

which, over a field, composes with the Eilenberg-Zilber and Künneth isomorphisms

$$
\begin{gathered}
\operatorname{Cotor}^{C^{N}(X \times X)}(R, R) \stackrel{\operatorname{Cotor} \nabla(R, R)}{\longleftarrow} \operatorname{Cotor}^{C^{N}(X) \otimes C^{N}(X)}(R, R) \\
\cong \operatorname{Cotor}^{C^{N}(X)}(R, R) \otimes \operatorname{Cotor}^{C^{N}(X)}(R, R)
\end{gathered}
$$

to give a coalgebra structure compatible with geometry. With the normalized chains the Eilenberg-Zilber map is both an equivalence and a map of differential coalgbras. Hence, it induces a map of Cotors. The Alexander-Whitney map

$$
C^{N}(X \times X) \stackrel{\Delta}{\rightarrow} C^{N}(X) \otimes C^{N}(X)
$$

is not a map of differential coalgebras and hence cannot be used to induce a map of Cotors.

Recall that Quillen constructed a functor from simply connected 1-reduced simplicial sets to simply connected commutative differential coalgebras over the rationals, $X \mapsto C^{Q}(X)$ having the properties that there is a natural isomorphism of coalgebras $H(X ; Q) \cong H C^{Q}(X)$ and a natural isomorphism of Lie algebras $(\pi \Omega X) \otimes Q \cong$ $H L\left(s^{-1} \bar{C}^{Q}(X)\right)$. 
We can substitute Quillen's commutative cochains $C^{Q}(X)$ for the normalized chains $C^{N}(X)$ and change the way in which $H \Omega C^{Q}(X)$ is seen to be a coalgebra (and thus a Hopf algebra). We use the Alexander-Whitney map $C^{Q} X \stackrel{\Delta}{\rightarrow} C^{Q}(X) \otimes C^{Q}(X)$ which now is a map of differential coalgebras. In fact, it is the unique map of commutative differential coalgebras which projects to the identities on both factors.

Just as before, the diagonal in the loop space $\Omega X \rightarrow \Omega X \times \Omega X$ is represented in homology by the map of differential Cotors

$$
\operatorname{Cotor}^{\Delta}(Q, Q): \operatorname{Cotor}^{C_{(X)}}(Q, Q) \rightarrow \operatorname{Cotor}^{C^{Q}(X \times X)}(Q, Q) .
$$

(This requires that Quillen's chain functor gives a Serre spectral sequence, 10,16.2. 10.21.1 [23], to fibrations. And it does.) But now, since the Alexander-Whitney map is a map of differential coalgebras, we can compose with the isomorphisms

$$
\begin{gathered}
\operatorname{Cotor}^{C_{(X \times X)}}(Q, Q) \stackrel{\operatorname{Cotor}^{\Delta}(Q, Q)}{\longrightarrow} \operatorname{Cotor}^{C_{(X) o \times C} C_{(X)}}(Q, Q) \\
\cong \operatorname{Cotor}^{C_{(X)}}(Q, Q) \otimes \operatorname{Cotor}^{C_{(X)}}(Q, Q)
\end{gathered}
$$

to give a coalgebra structure which is isomorphic to that in the rational homology of the loop space.

This coalgebra structure "covers" ( is induced by) the Alexander-Whitney map of differential coalgebras on the base

$$
C^{Q} X \stackrel{\Delta}{\rightarrow} C^{Q} X \otimes C^{Q} X
$$

Hence, Corollary 10.6 applies to show that the diagonal in the rational homology of the loop space

$$
\Delta: H \Omega X ; Q) \rightarrow H \Omega X ; Q) \otimes H \Omega X ; Q)
$$

is given by that in

$$
H \Omega C^{Q}(X)=\operatorname{Cotor}^{C^{Q}(X)}(Q, Q)
$$

which is a primitively generated Hopf algebra.

By the Poincare Birkhoff Witt theorem [19-21], there is an isomorphism of differential coalgebras $U L \cong S L$ where $S L$ is the free commutative algebra generated by the differential vector space which is the connected Lie algebra $L$. It follows that there are isomorphisms

$$
H U L \cong H S L \cong S H L \cong U H L
$$

Since $\Omega C^{Q}(X)=U L\left(s^{-1} \bar{C}^{Q}(X)\right)$ is primitively generated and there are natural isomorphisms of Hopf algebras 


$$
\begin{aligned}
H(\Omega X ; Q) & \cong H \Omega C^{Q}(X) \cong H U L\left(s^{-1} \bar{C}^{Q}(X)\right) \\
& \cong U H L\left(s^{-1} \bar{C}^{Q}(X)\right) \cong U(\pi \Omega X \otimes Q) .
\end{aligned}
$$

Quillen's methods have given another proof of the Milnor-Moore result, at least for loop spaces.

Hence, if $\mathrm{X}$ is simply connected, then $H \Omega C^{Q}(X) \cong H \Omega X$ is an isomorphism of primitively generated rational Hopf algebras. Therefore, in contrast to the integral case where we do not have commutative cochains, we see that there is a natural Hopf algebra structure on the rational Hopf algebra $H \Omega C^{Q}(X)$ which is both invariant under homological equivalence of the commutative coalgebra $C$ and which agrees with the structure on the rational homology Hopf algebra of the loop space.

\section{Quillen's model category of commutative simply connected rational differential graded coalgebras}

When doing rational homotopy theory, it is sometimes better to forget about spaces and instead focus directly on one of the homotopy equivalent model categories, for example, Quillen's model category $\mathcal{C}$ of commutative simply connected differential graded coalgebras over the rationals [25]. This model category structure consists of homology isomorphisms as weak equivalences, injections as cofibrations, and sufficient fibrations are given by Cartan's constructions.

We remark that, if the ground ring is a field $k$ of finite characteristic, the above does not provide a model category structure. Merely consider the dual problem of commutative differential graded algebras. It is easy to see that one cannot factor $k \rightarrow A \stackrel{p}{\rightarrow} P[x]$ where $A$ is acyclic, $p$ is an epimorphism, and $x$ is a generator of degree 2 .

Quillen constructs his fibrations with reference to the category of connected differential graded Lie algebras $\mathcal{L}$ over the rationals and his cofree coalgebra functor $C: \mathcal{L} \rightarrow \mathcal{C}$. The differential in $C(L)=S(s L)$ (= the symmetric coalgebra) is a sum of two parts, first, the suspension of that from $L$ and, second, a part coming from the Lie bracket of $L$. The second part is the dual of a construction due to Koszul [17]. For more details see [25].

Quillen obtains enough fibrations $E \rightarrow B$ by starting with a fibration in $\mathcal{L}$, that is, with a short exact sequence of differential graded Lie algebras

$$
0 \rightarrow L_{3} \rightarrow L_{2} \rightarrow L_{1} \rightarrow 0
$$

and then forming pullback diagrams

$$
\begin{aligned}
& E \rightarrow C\left(L_{2}\right) \\
& \downarrow \\
& B \rightarrow C\left(L_{1}\right) .
\end{aligned}
$$

As a differential comodule

$$
C\left(L_{2}\right)=C\left(L_{1}\right) \otimes C\left(L_{3}\right)
$$


over the "base" differential coalgebra $C\left(L_{1}\right)$ with "fibre" the subcomplex $C\left(L_{3}\right)$. That is, $C\left(L_{2}\right)$ is a Cartan construction [23] over $C\left(L_{1}\right)$ with fibre $C\left(L_{3}\right)$. Hence, the fibration $E \rightarrow B$ is a Cartan construction over $B$ with fibre $C\left(L_{3}\right)$, that is, $E=B \otimes C\left(L_{3}\right)$ as a $B$ differential comodule with $C\left(L_{3}\right)$ a subcomplex. We have used left constructions but we could have used right constructions if we so desired.

Theorem 12.1 In the category $\mathcal{C}$, the cotensor product gives a pullback diagram

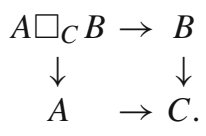

We need to show three things:

1. $A \square_{C} B$ is a commutative coalgebra.

2. the diagram is a commutative diagram of coalgebras.

3. it has the universal mapping property.

First of all, if $f: X \rightarrow A$ and $g: X \rightarrow B$ are maps of commutative differential graded coalgebras which are equal when projected to $C$, then $X \stackrel{\Delta}{\rightarrow} X \otimes X \stackrel{f \otimes g}{\longrightarrow} A \otimes B$ is a map of differential coalgebras which projects to $f$ and $g$. Since $\operatorname{map}(X, A \otimes B)=$ $\operatorname{map} X, P(A \otimes B))=\operatorname{map}(X, P(A) \oplus P(B))$ is determined by projection to the primitives, it is the unique such map.

On the other hand, since $\operatorname{map}(X, A \otimes C \otimes B)=\operatorname{map}(X, P(A) \oplus P(C) \oplus P(B))$, the above map factors through $A \square_{C} B$. It is therefore sufficient to know that $A \square_{C} B \subseteq$ $A \otimes B$ is a subcoalgebra.

It is instructive to consider the dual situation. Suppose $f, g: X \rightarrow Y$ are two maps of commutative differential graded algebras. The linear coequalizer of these maps is $Y /\{f x-g x \mid x \in X\}$. The algebra coequalizer is $Y /\{y(f x-g x) \mid x \in X, y \in Y\}$. Clearly, if there is a linear map $h: Y \rightarrow X$ which is a common section to both, $f h=g h=1_{Y}$, then the linear and algebra coequalizers are the same.

The dual is:

Lemma 12.2 Suppose $f, g: A \rightarrow B$ are two maps of commutative differential graded algebras. If there is a linear map $k: B \rightarrow A$ which is a common retraction to both, $k f=k g=1_{A}$, show that the linear equalizer $\operatorname{ker}(f-g): A \rightarrow B$ is the same as the coalgebra equalizer.

Proof Using $k$ is easy to see that $\Delta(\operatorname{ker}(f-g)) \subseteq \operatorname{ker}(f-g) \otimes A$. Similarly, $\Delta(\operatorname{ker}(f-g)) \subseteq A \otimes \operatorname{ker}(f-g)$ and thus $\Delta(\operatorname{ker}(f-g)) \subseteq \operatorname{ker}(f-g) \otimes \operatorname{ker}(f-g)$. Hence $\operatorname{ker}(f-g) \subseteq A$ is a subcoalgebra. That is, the linear equalizer is a subcoalgebra and therefore is the coalgebra equalizer.

In particular, the linear equalizer

$$
A \square_{C} B \subseteq A \otimes B \stackrel{1 \otimes \Delta, \Delta \otimes 1}{\longrightarrow} A \otimes C \otimes B
$$

is a subcoalgebra and hence is the coalgebra equalizer (since there is a common retraction $1_{A} \otimes \epsilon \otimes 1_{B}$ where $\epsilon: C \rightarrow Q$ is the counit). 
The Eilenberg-Moore approximation theorem is exactly Corollary 10.14.4 of [23].

\section{Theorem 12.3 If}

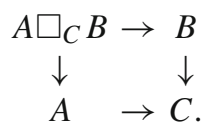

is a diagram with $A \rightarrow C$ a right Cartan construction (fibration) over $C$, then there is an isomorphism

$$
H\left(A \square_{C} B\right) \cong \operatorname{Cotor}^{C}(A, B) .
$$

There is a corresponding version of the above theorem for pullbacks of several variables [23]. In short, we have everything we need to study the multiplication and comultiplication in the loop space $\Omega C$. (We work over the rationals but we observe that the above three results are true over any field.)

The map in Corollary 10.6 defines a commutative differential coalgebra structures on $C \otimes_{\tau} \Omega C$ and on $\Omega C \otimes_{\tau} C$. Hence, these are suitable models for the path space fibrations and, in particular, $\Omega C=Q \square_{C} C \otimes_{\tau} \Omega C=\Omega C \otimes_{\tau} C \square_{C} Q$ is a model for the loop space.

Corollary 10.6 identifies the coalgebra structure in $\Omega C$ as the primitively generated one and the equivalence $\Omega C \otimes_{\tau} C \otimes_{\tau} C \Omega C \rightarrow \Omega C$ identifies the multiplicative structure with the tensor multiplication just as in section 7. In particular, $H \Omega C=$ $H T\left[s^{-1} \bar{C}\right]=H U L\left(s^{-1} \bar{C}\right)=U H L\left(s^{-1} \bar{C}\right)=\operatorname{Cotor}^{C}(Q, Q)$ as a primitively generated Hopf algebra.

\section{Homology of the double loops on spheres}

Since simplicial methods are not often amenable to computation, there is often no direct way of using the Eilenberg-Moore methods to compute the coalgebra structure in the homology of loop spaces. There are sometimes ways to get around this.

The easiest example is already given by the Bott-Samelson theorem. If $X$ is a connected space and the homology $H X$ is a free module, then there is a coalgebra equivalence $H \Sigma X \rightarrow C \Sigma X$ and an isomorphism of tensor algebras

$$
T(\bar{H} X) \rightarrow H \Omega \Sigma X \rightarrow
$$

with the coalgebra structure determined by the fact that $X \rightarrow \Omega \Sigma X$ induces a map of homology coalgebras.

We want to close with a more complicated example. This computation was originally done by different means, using the Serre spectral sequence, independently by Moore and Toda $[22,26]$. Here we use it to illustrate how the Eilenberg-Moore methods can be used to compute without the use of a spectral sequence, except for one use of the Serre integral cohomology spectral sequence to compute the Bockstein differentials. 
Theorem 13.1 (a) If $p$ is an odd prime, then the mod p homology of the double loop space of an odd sphere is

$$
H \Omega^{2} S^{2 n+1} \stackrel{\simeq}{\rightarrow} E\left(u, \tau_{1}, \tau_{2}, \ldots\right) \otimes P\left(\sigma_{1}, \sigma_{2}, \ldots\right)
$$

as a primitively generated Hopf algebra with

$$
\operatorname{deg}(u)=2 n-1, \quad \operatorname{deg}\left(\tau_{k}\right)=2 p^{k} n-1, \quad \operatorname{deg}\left(\sigma_{k}\right)=2 p^{k} n-2 .
$$

Furthermore, the first Bockstein differential is given by

$$
\beta^{1} u=0, \quad \beta^{1} \tau_{k}=\sigma_{k}, \quad \beta^{1} \sigma_{k}=0 .
$$

(b) If $p=2$, then the mod 2 homology of the double loop space of a sphere is

$$
H \Omega^{2} S^{m+1} \stackrel{\simeq}{\rightarrow} P\left(\xi_{0}, \xi_{1}, \xi_{2}, \ldots\right)
$$

as a primitively generated Hopf algebra with deg $\left(\xi_{k}\right)=2^{k} m-1$. Furthermore, if $m$ is even, the first Bockstein differential is given by $\beta^{1} \xi_{0}=0$ and $\beta^{1} \xi_{k}=\left(\xi_{k-1}\right)^{2}$ for $k \geq 1$.

Remark If $p$ is any prime, the even dimensional case reduces to the odd dimensional case via the following isomorphism [23] of primitively generated mod $p$ Hopf algebras

$$
H\left(\Omega^{2} S^{2 n+2}\right)=H\left(\Omega S^{2 n+1}\right) \otimes H\left(\Omega S^{4 n+3}\right) .
$$

This isomorphism is also compatible with the Bockstein differentials.

If $p$ is an odd prime, then this follows from Serre's splitting of spaces localized at $p$

$$
\Omega S^{2 n+2} \simeq S^{2 n+1} \times \Omega S^{4 n+3}
$$

Unless $n=1,2$, or 3, this splitting is not valid localized at 2. We have only the totally nonhomologous to zero fibration sequence given by the second Hopf invariant

$$
S^{2 n+1} \rightarrow \Omega S^{2 n+2} \stackrel{h_{2}}{\rightarrow} \Omega S^{4 n+3}
$$

But 12.5 below says that the loop of this sequence is also totally nonhomologous to zero and 12.6 shows that $H \Omega^{2} S^{2 n+2}$ is primitively generated. The Bockstein differentials are completely determined by their naturality with respect to the maps in the fibration sequence.

Hence, the even dimensional case reduces to the odd dimension case whenever the prime is even or odd.

The first thing we need to do is to show we can use homology instead of chains. Hence, 
Theorem 13.2 With any coefficients, there is a homology equivalence of differential coalgebras

$$
T[x]=H \Omega S^{m+1} \stackrel{f}{\rightarrow} C \Omega S^{m+1}
$$

where $\operatorname{deg}(x)=m$.

Proof We use here the loop space with the strictly associative multiplication so that the chains $C \Omega S^{m+1}$ are an associative differential Hopf algebra. Since $\Omega S^{m+1}$ is $m-1$ connected, the chains are equivalent to those from the Eilenberg subcomplex where all simplices are restricted to having their $m-1$ skeleton at the basepoint. If we choose a cycle $c$ from this subcomplex which represents the $m$ dimensional homology generator, then it is certainly primitive. Hence, the map defined by $f\left(x^{i}\right)=c^{i}$ is the required homology equivalence of differential coalgebras.

Therefore,

Theorem 13.3 With any coefficient ring $R$,

$$
H \Omega^{2} S^{m+1}=\operatorname{Cotor}^{T[x]}(R, R)=H \Omega T[x]
$$

as algebras.

If $x$ has even degree, then $T[x]$ is a commutative coalgebra, It follows that, over a field $\left.R, \operatorname{Cotor}^{T[x]} R, R\right)=H \Omega T[x]$ is actually a primitively generated Hopf algebra but it is possible that the coalgebra structures in the above theorem are different. The same remark holds if $x$ has odd degree and the field has characteristic 2 .

If $p$ is odd, assume that $\operatorname{deg}(x)=m=2 n$ is even. Then the tensor Hopf algebra $T[x]$ splits as a coalgebra into tensor product of coalgebras

$$
T[x]=\bigotimes_{i \geq 0} T_{p}\left[x^{p^{i}}\right]
$$

where

$$
T_{p}\left[x^{p^{i}}\right]=<1, x^{p^{i}}, x^{2 p^{i}}, \ldots, x^{(p-1) p^{i}}>
$$

If $p=2$, then $\operatorname{deg}(x)=m$ can be either even or odd and, In this case, the tensor Hopf algebra $T[x]$ splits as a coalgebra into

$$
T[x]=\bigotimes_{i \geq 0} T_{2}\left[x^{2^{i}}\right]
$$

where $T_{2}\left[x^{2^{i}}\right]=E\left[x^{2^{i}}\right]=<1, x^{2^{i}}>$ is the exterior coalgebra.

The Künneth theorem yields 
Theorem 13.4 Let $p$ be a prime and let $T[x]$ is a tensor algebra on a generator $x$ of degree $m$. Assume either that $m$ is even or that $p=2$, Then there is an isomorphism of primitively generated Hopf algebras

$$
\operatorname{Cotor}^{T[x]}(Z / p Z, Z / p Z)=\bigotimes_{i \geq 0} \operatorname{Cotor}^{T_{p}\left[x^{p^{i}}\right]}(Z / p Z, Z / p Z)
$$

We now compute the above Cotors:

Theorem 13.5 (Lemma) As primitively generated Hopf algebras:

(a) If $p$ is an odd prime and $T_{p}[x]$ is the coalgebra $<1, x, x^{2}, \ldots, x^{p-1}>$ with $x$ of even degree $2 n$, then

$$
\operatorname{Cotor}^{T_{p}[x]}(Z / p Z, Z / p Z)=E\left(s^{-1} x\right) \otimes P(z)
$$

where $\operatorname{deg}\left(s^{-1} x\right)=2 n-1, \operatorname{deg}(z)=2 p n-2$.

(b) If $p=2$ and $x$ has arbitrary degree $m$, then

$$
\operatorname{Cotor}^{E[x]}(Z / 2 Z, Z / 2 Z)=P\left(s^{-1} x\right)
$$

where $\operatorname{deg}\left(s^{-1} x\right)=m-1$.

We proceed to prove this lemma using duality.

Proof If $C$ is a finite type commutative coalgebra which is free over $R$, then the dual $A=C^{*}$ is a commutative algebra and we have that $\operatorname{Tor}_{A}(R, R)$ is a Hopf algebra which is dual to the Hopf algebra $\operatorname{Cotor}^{C}(R, R)$. If we use $Z_{(p)}$ coefficients and set $C=T_{p}(x)$ we get that $A=P_{p}(y)=P(y) /\left(y^{p}\right)=$ the truncated polynomial algebra with $\operatorname{deg}(y)=\operatorname{deg}(x)$.

With $p$ an odd prime and $Z_{(p)}$ coefficients, consider the complex

$$
\mathcal{R}=A \otimes E(s) \otimes \Gamma(t)
$$

with $\operatorname{deg}(s)=\operatorname{deg}(x)-1, \operatorname{deg}(t)=p \operatorname{deg}(x)$ and differential the derivation defined by

$$
d y=0, \quad d s=y, d t=s y^{p-1} .
$$

$\mathcal{R}$ is an acyclic complex and a differential algebra. It provides a resolution of $Z_{(p)}$ over the algebra $A$ and the multiplication is a map of resolutions

$$
\mathcal{R} \otimes \mathcal{R} \rightarrow \mathcal{R}
$$

and induces a multiplication on

$$
\mathcal{R} \otimes_{A} Z_{(p)}=E(s) \otimes \Gamma(t) .
$$


Since the latter has zero differential,

$$
\operatorname{Tor}_{A}\left(Z_{(p)}, Z_{(p)}\right)=E(s) \otimes \Gamma(t)
$$

as algebras.

If $p$ is odd, both the elements $s$ and $t$ must be primitive for degree reasons. Hence, in this case, we have

$$
\operatorname{Tor}_{A}\left(Z_{(p)}, Z_{(p)}\right)=E(s) \otimes \Gamma(t)
$$

as Hopf algebras. It follows that

$$
\operatorname{Cotor}^{T_{p}(x)}\left(Z_{(p)}, Z_{(p)}\right)=E\left(s^{-1} x\right) \otimes P(z)
$$

as Hopf algebras. Of course, we can reduce this result mod $p$ to the desired result with $Z / p Z$ coefficients.

If $p=2$, consider the complex over the ring $Z / 2 Z$ given by

$$
\mathcal{R}=A \otimes \Gamma[s]
$$

with $\operatorname{deg}(s)=\operatorname{deg}(x)-1$ and with the derivation defined by

$$
d y=0, d \gamma_{k}(s)=y \gamma_{k-1}(s)
$$

In particular, we have

$$
d \gamma_{1}(s)=d s=y \gamma_{0}(s)=y, \quad d \gamma_{2}(s)=y \gamma_{1}(s)=y s .
$$

It is an acyclic differential Hopf algebra. In particular, both the multiplication and the comultiplication

$$
\mathcal{R} \otimes \mathcal{R} \rightarrow \mathcal{R}, \quad \mathcal{R} \rightarrow \mathcal{R} \otimes \mathcal{R}
$$

are maps of resolutions. Note that $y$ and $s$ are primitive but

$$
\Delta\left(\gamma_{2}(s)\right)=\gamma_{2}(s) \otimes 1+s \otimes s+1 \otimes \gamma_{2}(s)
$$

This is dual to the statement that the square of the 1 dimensional class is the 2 dimensional class.

It follows that

$$
\operatorname{Tor}_{A}(Z / 2 Z, Z / 2 Z)=\Gamma[s]
$$

as a Hopf algebra.

Hence,

$$
\operatorname{Cotor}^{T_{2}(x)}(Z / 2 Z, Z / 2 Z)=P\left(s^{-1} x\right)
$$

as a Hopf algebra. 
We conclude that we have proved the algebra part of 12.1 .

Theorem 13.6 Let $p$ be a prime.

(a) If $p$ is odd, there is an isomorphism of algebras

$$
H\left(\Omega^{2} S^{2 n+1} ; Z / p Z\right)=E\left(u, \tau_{1}, \tau_{2}, \ldots\right) \otimes P\left(\sigma_{1}, \sigma_{2}, \ldots\right)
$$

where $\operatorname{deg}(u)=2 n-1, \operatorname{deg}\left(\tau_{i}\right)=2 p^{i} n-1$, and $\operatorname{deg}\left(\sigma_{i}\right)=2 p^{i} n-2$.

(b) If $p=2$, there is an isomorphism of algebras

$$
H\left(\Omega^{2} S^{m+1} ; Z / 2 Z\right)=P\left(\xi_{0}, \xi_{2}, \ldots\right)
$$

where $\operatorname{deg}\left(\xi_{i}\right)=2^{i} m-1$.

That the results in 12.6 are isomorphisms of primitively generated Hopf algebras follows from the dual of a result of Milnor and Moore [20,21].

Theorem 13.7 Suppose that B is a connected Hopf algebra over $Z / p Z$ with commutative multiplication. Let $\xi: B \rightarrow B$ be the $p$-th power operation $\xi(x)=x^{p}$ and consider the subHopf algebra $\xi B \subseteq B$ of all $p$-th powers. There is an exact sequence

$$
0 \rightarrow P(\xi B) \rightarrow P(B) \rightarrow Q(B)
$$

In particular, the kernel of the map $P(B) \rightarrow Q(B)$ is concentrated in degrees divisible by $p$.

The dual to the above says, if $D$ is a connected Hopf algebra over $Z / p Z$ with commutative comultiplication, then the cokernel of $P(D) \rightarrow Q(D)$ is concentrated in degrees divisible by $p$. Hence, the Hopf algebras in 12.6 are primitively generated and isomorphic as Hopf algebras.

We complete the proof of 12.1 by determining the first Bockstein differentials $\beta^{1}$. We start with an explicit computation over $Z_{(p)}$.

Theorem 13.8 For all primes $p$,

$$
H_{2 p n-2}\left(\Omega^{2} S^{2 n+1} ; Z_{(p)}\right)=Z / p Z .
$$

Proof Consider the $Z_{(p)}$ cohomology Serre spectral sequence of the path space fibration sequence

$$
\Omega^{2} S^{2 n+1} \rightarrow P \Omega S^{2 n+1} \rightarrow \Omega S^{2 n+1}
$$

Since the $Z_{(p)}$ cohomology of the base is the divided power algebra $H^{*}\left(\Omega S^{2 n+1} ; Z_{(p)}\right)$ $=\Gamma[y]$ with $\operatorname{deg}(y)=2 n$, the classic argument shows that

$$
H^{2 p n-2}\left(\Omega^{2} S^{2 n+1} ; Z_{(p)}\right)=0, \quad H^{2 p n-1}\left(\Omega^{2} S^{2 n+1} ; Z_{(p)}\right)=Z / p Z
$$

and hence $H_{2 p n-2}\left(\Omega^{2} S^{2 n+1} ; Z_{(p)}\right)=Z / p Z$. 
It follows that we must have

(a) if $p$ is an odd prime, then $\beta^{1} \tau_{1}=\sigma_{1}$ in dimension $2 p n-2$.

(b) if $p=2$, then $\beta^{1} \xi_{1}=\left(\xi_{0}\right)^{2}$.

Now consider the $p$-th Hopf invariant $h_{p}: \Omega S^{2 n+1} \rightarrow \Omega S^{2 p n+1}$. Localized at $p$, we have a fibration sequence which is totally nonhomologous to zero

$$
F \rightarrow \Omega S^{2 n+1} \stackrel{h_{p}}{\rightarrow} \Omega S^{2 p n+1}
$$

where $H F=T_{p}\left[x_{2 n}\right]$ the Hopf invariant induces the map

$h_{p}: H \Omega S^{2 n+1}=T\left[x_{2 n}\right]=\bigotimes_{i \geq 0} T_{p}\left[x_{2 n}^{p^{i}}\right] \rightarrow H \Omega S^{2 p n+1}=T\left[x_{2 p n}\right]=\bigotimes_{i \geq 0} T_{p}\left[x_{2 p n}^{p^{i}}\right]$

such that for $i \geq 1$

$$
h_{p}\left(T_{p}\left[x_{2 n}^{p^{i}}\right]\right)=T_{p}\left[x_{2 p n}^{p^{i-1}}\right] .
$$

It follows that the loop

$$
\Omega h_{p}: \Omega^{2} S^{2 n+1} \rightarrow \Omega^{2} S^{2 p n+1}
$$

satisfies, for $i \geq 2$, up to units,

(a) if $p$ is odd,

$$
\Omega h_{p}\left(\tau_{i}(2 n)=\tau_{i-1}(2 n p), \quad \Omega h_{p}\left(\sigma_{i}(2 n)\right)=\sigma_{i-1}(2 n p)\right.
$$

(b) and if $p=2$,

$$
\Omega h_{2}\left(\xi_{i}(2 n)\right)=\xi_{i-1}(4 n), \quad \Omega h_{2}\left(\xi_{i-1}(2 n)\right)^{2}=\left(\xi_{i-1}(4 n)\right)^{2} .
$$

Hence, whenever we know the formula for $\beta^{1} \tau_{i}(2 n)$, we know the formula for $\beta^{1} \tau_{i}(2 n p)$, and naturality gives the formula for $\beta^{1} \tau_{i+1}(2 n)$. And similarly for $\beta^{1} \xi(2 n)$. Hence, induction shows

Theorem 13.9 For all $i \geq 1, \beta^{1} \tau_{i}=\sigma_{i}$ if $p$ is an odd prime and $\beta^{1} \xi_{i}=\left(\xi_{i-1}\right)^{2}$ if $p=2$.

Since the first Bockstein makes the mod $p$ homology acyclic except in the bottom nontrivial dimensions, we get the following corollary which is crucial in the study of the odd primary exponents of the homotopy groups of spheres and Moore spaces. $[7,23]$

Theorem 13.10 For any prime $p$, the homology localized at $p$ of $\Omega^{2} S^{2 n+1}$ has a $Z_{(p)}$ in dimension $2 n-1$ and torsion of order at most $p$ in all higher dimensions. 


\section{Closing remarks on the Hopf algebra structure in the cobar construction}

When $X$ is a simply connected space, then, over a field, $H \Omega C(X)$ is a Hopf algebra. At first, it seems that the comultiplication is more fundamental since it is induced by the diagonal map of spaces $X \rightarrow X \times X$ while the multiplication is induced by a map $X \times X \rightarrow X$ only when $X$ is an $\mathrm{H}$ space.

In fact, the multiplication is more fundamental since $H \Omega C$ has a multiplication whenever $C$ is a differential coalgebra. Although we do not show it here, this multiplication is like the multiplication in the fundamental group of a loop space in the following sense. In the presence of another multiplication, for example, when $C$ is a differential Hopf algebra (and the multiplication $C \otimes C \rightarrow C$ is a map of differential coalgebras), the resulting two multiplications on $H \Omega C$ agree and both are (graded) commutative [23].

When $C$ is not a commutative coalgebra, $H \Omega C$ may not have a comultiplication since the diagonal map $C \rightarrow C \otimes C$ is not a map of differential coalgebras. Even when $C$ is a commutative coalgebra, the resulting comultiplication on $H \Omega C$, which over a field is always primitively generated, may have little to do with geometry.

Over a field, $H \Omega C(X)$ is a coalgebra (and thus a Hopf algebra) by virtue of the map $C X \rightarrow C(X \times X)$ and the Eilenberg Zilber equivalence $C X \otimes C X \rightarrow C(X \times X)$. It has little to do with the Alexander Whitney map $C X \rightarrow C X \otimes C X$ since this is not a map of coalgebras. But, most important, this coalgebra structure in $H \Omega C(X)$ does correspond to the comultiplication in the homology of the loop space since it corresponds to the diagonal in the loop space.

The ambiguity in the coalgebra structures on $H \Omega C(X)$ disappears when we have rational coefficients. They are the same and both are primitively generated.

\section{References}

1. Adams, J.F.: On the cobar construction. Proc. Natl. Acad. Sci. USA 42, 409-412 (1956)

2. Adams, J.F., Hilton, P.J.: On the chain algebra of a loop space. Commun. Math. Helv. 30, 305-330 (1956)

3. Baues, H.-J.: The cobar construction as a Hopf algebra. Invent. Math. 132, 467-489 (1998)

4. Bott, R., Samelson, H.: On the Pontrjagin product in spaces of paths. Comment. Math. Helv. 27, 320-337 (1953)

5. Browder, W.: On differential Hopf algebras. Trans. Am. Math. Soc. 107, 153-176 (1963)

6. Cartan, H.: Algebres d'Eilenberg-MacLane, Seminaire Henri Cartan 1954/55, exposes 2-11. Ecole Normal Supérieure, Paris (1955)

7. Cohen, F.R., Moore, J.C., Neisendorfer, J.A.: Torsion in homotopy groups. Ann. Math. 109, 121-168 (1979)

8. Eilenberg, S., Moore, J.C.: Adjoint functors and triples. Ill. J. Math. 9, 381-398 (1965)

9. Eilenberg, S., Moore, J.C.: Homology and fibrations i, coalgebras, cotensor product and its derived functors. Comment. Math. Helv. 40, 199-236 (1966)

10. Getzler, E., Goerss, P.G.: A model category structure for differential graded coalgebras (1999). (preprint)

11. Goerss, P.G., Jardine, J.F.: Simplicial Homotopy Theory. Birkhauser, Basel (1999)

12. Hess, K.: Homotopic Hopf-Galois extensions: foundations and examples. Geom. Topol. Monogr. 16, 79-132 (2009) 
13. Hess, K., Kedziorak, M., Riehl, E., Shipley, B.: A necessary and sufficient condition for induced model structures (2015). (preprint)

14. Hess, K., Parent, P.-E., Scott, J., Tonks, A.: A canonical enriched Adams-Hilton model for simplicial sets. Adv. Math. 207(2), 847-875 (2005)

15. Hess, K., Shipley, B.: The homotopy theory of coalgebras over a comonad. Proc. London Math. Soc. 108(2), 484-516 (2014)

16. Husemoller, D., Moore, J.C., Stasheff, J.D.: Differential homological algebra and homogeneous spaces. J. Pure Appl. Alg. 5, 113-185 (1974)

17. Koszul, J.L.: Homologie et cohomology des algebres de Lie. Bull. Soc. Math. France 85, 239-262 (1957)

18. MacLane, S.: Homology. Springer, Berlin (1963)

19. May, J.P., Ponto, K.: More Concise Algebraic Topology. University of Chicago, Chicago (2012)

20. Milnor, J.W., Moore, J.C.: On the structure of Hopf algebras. Ann. Math. 81, 211-264 (1965)

21. Milnor, J.W., Moore, J.C.: On the structure of Hopf algebras (preprint 1959). In: Bass, H., Lam, T.Y. (eds.) Collected papers of John Milnor, V, Algebra, pp. 7-36. Amer. Math. Soc., USA (2011)

22. Moore, J.C.: The double suspension and p-primary components of the homotopy groups of spheres. Boll. Soc. Mat. Mexicana 1, 28-37 (1956)

23. Neisendorfer, J.A.: Algebraic Methods in Unstable Homotopy Theory. Cambridge University Press, Cambridge (2009)

24. Quillen, D.: Homotopical Algebra. Springer, Berlin (1967)

25. Quillen, D.: Rational homotopy theory. Ann. Math. 90, 295-295 (1969)

26. Toda, H.: On the double suspension $E^{2}$. J. Inst. Polytech. Osaka City Univ. Ser. A 7, 103-145 (1956) 